\title{
Gene expression stability at high evolutionary distances
}

\author{
Klepikova A.V.*, Kasianov A.S., Penin A.A. \\ ${ }^{1}$ Institute for Information Transmission Problems RAS, Moscow, Russia \\ * email: annklepikova@gmail.com
}

Stably expressed (stable) genes are the genes with approximately uniform expression level across various organs and tissues of the organism and/or different conditions. Stably expressed genes were thoroughly analyzed for a plenty of both model and nonmodel species using large-scale data including single-celled RNA-seq. The primary motivation for stable genes studies is usually the identification of reference genes for normalization of gene expression level between samples in real-time qPCR and RNAseq. On the other hand, the interesting questions are which biological functions need a uniform amount of genes mRNA in the whole organism and are the expression stability evolutionary conservative between distantly related species. To address these questions, we analyzed transcriptome maps of five plant species: Arabidopsis thaliana, Fagopyrum esculentum (buckwheat), Phalaenopsis equestris, Solanum lycopersicum (tomato), and Zea mays (maize). We found stably expressed genes for every species and studied their orthologization. Genes that were stable in all species participated in RNA splicing and modifications, membrane organization, transport and mitochondria functioning. We analyzed the regulation of stably expressed genes and identified conditions that disturb their expression level. Our results suggest an explanation of the conservativeness of gene expression stability at high evolutionary distances.

Acknowledgements: The reported study was funded by RFBR according to the research project No. 18-29-13017. 